\title{
LETTERS
}

\section{Role of dietitians in reducing malnutrition in hospital}

Often when we think of malnutrition, we think of extreme examples, such as starving children in developing nations, or emaciated individuals in war-torn areas and World War II concentration camps. However, these extreme images are relatively rare, and malnutrition is not always visible to the naked eye of health care professionals who are not trained to identify this condition. In Canada, an estimated $45 \%$ of medical and surgical patients admitted to hospital are identified as malnourished. ${ }^{1}$ In hospital, patients can become malnourished very quickly. Patients admitted to hospital often miss multiple meals during their hospital stays owing to being kept on "nothing by mouth" instructions for medical tests and procedures, or feeling unwell with concurrent anorexia. Combined with a lack of physical activity, patients admitted to hospital begin to lose muscle mass at a rate of $0.5 \%$ of total body muscle mass per day, ${ }^{2}$ which can lead to substantial muscle loss during periods of immobility, ${ }^{3}$ as commonly experienced during a hospital stay.

Early nutrition intervention is key to curtailing malnutrition. Dietitians are health care professionals who undergo specialized training in nutrition to focus on assessing and identifying patients at risk of malnutrition, preventing malnutrition and improving nutrition status. As mentioned by Eckert and Cahill in their CMAJ article, ${ }^{4}$ an effective strategy for preventing malnutrition is to involve the dietitian early, within 24 hours of hospital admission. For patients at risk of malnutrition, dietitian assessment and intervention is effective in improving dietary intake and quality. ${ }^{4}$ When a dietitian is consulted, the dietitian will evaluate the patient and tailor the nutrition care plan to the individual's needs.

Some of the dietitian-led initiatives undertaken in hospitals across Canada include the Enhanced Recovery after Surgery (ERAS) protocol, the Medication Pass Nutrition Supplement Program (Med Pass), the Canadian Nutrition Screening Tool (CNST) and protected meal times.

Enhanced Recovery after Surgery ${ }^{5}$ is a program that has a goal to improve patient outcomes after surgery, including shortening length of hospital stay and reducing surgical complications. A large component of ERAS focuses on maintaining patients' nutrition status and decreasing risk of malnutrition by preventing preoperative fasting and promoting early postoperative feeding through the administration of oral nutrition supplements.

Med Pass $^{6}$ is another initiative that aims to decrease risk of malnutrition by having patients consume an oral nutrition supplement beverage with their medications, in place of water, to provide additional calories and protein.

The CNST, ${ }^{7}$ a tool recommended by the Canadian Malnutrition Task Force, is being implemented in many health care facilities as well. The purpose of the CNST is to identify patients who are at risk of malnutrition on admission to hospital (i.e., within the recommended 24 hours) and subsequently submit a referral to the dietitian for those patients.

Protected meal times are also being implemented to ensure that patients have sufficient time to eat their meals and are not interrupted by health care professionals or medical tests and procedures.

Physicians should feel comfortable referring their patients who are admitted to hospital to a dietitian for evaluation of malnutrition risk. Early intervention of malnutrition is important, and dietitians can use their professional expertise to attenuate this problem.

\section{Rebecca A. Holmes MSc RD}

Registered dietitian, Dietitians of Canada (member), Calgary, Alta.

Cite as: CMAJ 2019 February 4;191:E139. doi: $10.1503 / \mathrm{cmaj} .71130$

\section{References}

1. Allard JP, Keller H, Jeejeebhoy KN, et al. Malnutrition at hospital admission-contributors and effect on length of stay: a prospective cohort study from the Canadian Malnutrition Task Force. JPEN J Parenter Enteral Nutr 2016;40:487-97.

2. Wall BT, van Loon LJ. Nutritional strategies to attenuate muscle disuse atrophy. Nutr Rev 2013; 71:195-208.

3. Wall BT, Dirks ML, Snijders T, et al. Substantial skeletal muscle loss occurs during only 5 days of disuse. Acta Physiol (Oxf) 2014;210:600-11.

4. Eckert KF, Cahill LE. Malnutrition in Canadian hospitals. CMAJ 2018;190:E1207.

5. ERAS Society guidelines. Available: http://eras society.org/guidelines/list-of-guidelines (accessed 2018 Dec. 19).

6. Kerrigan ER, Maxwell J, Siegei C, et al. Dispensing of a calorically dense oral supplement with medication pass: a creative approach to oral supplementation. J Am Diet Assoc 1996;96(Suppl): A32.

7. Canadian Nutrition Screening Tool (CNST). Canadian Nutrition Society. Available: http://nutrition careincanada.ca/sites/default/uploads/files/CNST .pdf (accessed 2018 Dec. 19).

Competing interests: Rebecca Holmes is a member of Dietitians of Canada and is chair of the Dietitians of Canada Continuing Education Committee - Calgary. 